Revista Brasileira de Meteorologia, v.28, n.3, 257 - 266, 2013

\title{
ANÁLISE DA VELOCIDADE DO VENTO E ALTURA DE ONDA EM INCIDENTES DE NAUFRÁGIO NA COSTA BRASILEIRA ENTRE OS ESTADOS DO SERGIPE E DO RIO GRANDE DO SUL
}

\author{
EDUARDO VETROMILLA FUENTES ${ }^{1}$, DANIEL PIRES BITENCOURT ${ }^{2}$, MÁRCIA \\ VETROMILLA FUENTES ${ }^{3}$
}

\author{
${ }^{1}$ Universidade Federal de Santa Catarina, Departamento de Ecologia e Zoologia, Laboratório de Ecologia de \\ Águas Continentais, Florianópolis, SC, Brasil \\ ${ }^{2}$ Fundação Jorge Duprat Figueiredo de Segurança e Medicina do Trabalho (FUNDACENTRO), \\ Florianópolis, SC, Brasil \\ ${ }^{3}$ Instituto Federal de Santa Catarina (IFSC), Florianópolis, SC, Brasil
}

vetromilla@gmail.com,daniel@fundacentro.sc.gov.br,marciaf@ifsc.edu.br

Recebido Maio de 2011 - Aceito Fevereiro de 2013

\begin{abstract}
RESUMO
A ocorrência de naufrágios pode ser atribuída a vários fatores. Contudo, embarcações de pequeno porte usadas na pesca costeira, são geralmente mais suscetíveis aos fatores meteorológicos e oceânicos. O objetivo desse estudo foi analisar o comportamento da velocidade do vento e altura significativa de onda em relação à ocorrência de naufrágios na costa brasileira, entre os estados de Sergipe e Rio Grande do Sul. As frequências trimestral e mensal de naufrágios registrados entre Janeiro/2004 e Junho/2008 foram comparados com os ventos e com a altura significativa de ondas. As velocidades médias e máximas do vento nos dias e áreas de ocorrência, bem como a altura significativa de onda, foram comparadas aos valores históricos dessas variáveis. Foram observados ventos e ondas acima da climatologia em $54 \%$ e $46 \%$ das ocorrências de naufrágio, respectivamente. Em vários casos, o vento atingiu velocidades muito elevadas, sendo a provável causa para a ocorrência desses naufrágios. Os resultados apontaram para uma maior incidência de naufrágios em ambientes costeiros das regiões sudeste e sul do Brasil, com maior ocorrência entre os meses de março e agosto, envolvendo principalmente embarcações de pequeno porte (94\%), utilizadas na pesca costeira. Os resultados encontrados reforçam a necessidade do envio de informações meteorológicas e oceanográficas para pequenas embarcações, em especial àquelas empregadas na atividade de pesca costeira.

Palavras-chave: climatologia, embarcações de pequeno porte, pesca costeira, naufrágios, Brasil
\end{abstract}

\begin{abstract}
ANALYSIS OF WIND SPEED AND WAVE HEIGHT IN SHIPWRECK INCIDENTS ALONG THE BRAZILIAN COAST BETWEEN THE STATES OF SERGIPE AND RIO GRANDE DO SUL: Shipwrecks may occur due to several factors. However, small vessels used in coastal fisheries are more vulnerable to the weather and ocean conditions. The aim of this study is to analyze wind speed and significant wave height data during the shipwrecks recorded along the Brazilian coast, between the states of Sergipe and Rio Grande do Sul. Quarterly and monthly frequencies of shipwrecks recorded from January 2004 to June 2008 were compared to wind speeds as well as significant wave heights. The average and maximum speed of the wind at the days of the shipwrecks, as well as the significant wave height were compared to the historical data of these variables. Wind speeds and wave heights higher than the climatology were observed in about $54 \%$ and $46 \%$ of shipwreck occurrences, respectively. In several cases, the strong wind may have been the main cause for the shipwreck. Most shipwrecks occurred in the south and southeastern offshore regions between March and August, involving small vessels (94\%) used in coastal fisheries. The results reinforce the need to provide meteorological and oceanographic forecasts to small vessels, especially those employed in coastal fisheries.
\end{abstract}

Keywords: climatology, small vessels, coastal fisheries, shipwrecks, Brazil 


\section{INTRODUÇÃO}

A segurança no transporte e navegação marítima é uma das principais preocupações dos projetistas e construtores de embarcações (Soares e Teixeira, 2001). O naufrágio está entre as ocorrências mais perigosas para tripulantes e passageiros, e pode ser definido como a perda total ou parcial da embarcação por soçobramento. Pesquisas sobre causas de acidentes no mar se utilizam, em geral, de dados estatísticos referentes a incidentes com navios de grande porte (Lois et al., 2002; Toffoli et al., 2003). Em embarcações menores, diversas causas podem ser atribuídas, como incêndio, colisão, excesso ou má distribuição de carga, falta de manutenção em estruturas ou equipamentos da embarcação, imprudência de condutores e condições adversas de tempo e mar. Jin et al. (2001) mostraram que é esperado um número maior de vítimas fatais em casos de incêndio/explosão e naufrágio, podendo ocorrer, respectivamente, de 3,5 a 3,8 mortes a cada 100 acidentes.

Embarcações de pequeno a médio porte (i.e. embarcações utilizadas na pesca artesanal) são potencialmente mais propensas à ocorrência de naufrágio causado por condições adversas do clima. Ao analisarem sinistros em embarcações pesqueiras na região de Cabo Frio/RJ, Neves et al. (2002) perceberam forte sazonalidade na ocorrência de acidentes, sendo levados a supor que tais incidentes estiveram fortemente correlacionados às condições meteorológicas. Estudos de modelagem em segurança na navegação identificaram que o aumento da velocidade do vento eleva a probabilidade de acidentes no setor da pesca, que tende a ser maior para embarcações médias que navegam próximo à costa (Jin et al., 2002; Jin e Thunberg, 2005).

Os ciclones extratropicais são importantes sistemas meteorológicos, capazes de gerar tempestades com chuva e vento intensos. Esses sistemas atuam em todas as épocas do ano na costa leste da América do Sul, mas com maior frequência no inverno (Gan e Rao, 1991; Sinclair, 1994, 1995). No Atlântico Sudoeste, durante os meses de junho, julho e agosto, a densidade dos sistemas ciclônicos é de aproximadamente $1 \times 10^{-3}$ ciclones ${ }^{\circ} \mathrm{lat}^{-2}$ maior que nos demais meses (Simmonds e Keay, 2000). As frentes frias também atuam durante o ano todo. Porém, devido ao avanço das massas de ar frio, os gradientes horizontais de temperatura e pressão são mais pronunciados nos meses de inverno (Garreaud, 2000), sendo que, quanto maior o gradiente horizontal de pressão, mais intensos podem ser os ventos. Distúrbios transientes que se deslocam em altitude de oeste para leste, como os vórtices ciclônicos, costumam causar chuvas e ventos intensos na região oceânica da costa brasileira. Segundo Satyamurty et al. (1990), aproximadamente 100 vórtices ciclônicos atravessam anualmente o continente sul-americano, de oeste para leste, entre 15 e $60^{\circ} \mathrm{S}$ e, na maioria das vezes, geram ou intensificam tempestades na mesma faixa latitudinal, entre 30 e $70^{\circ} \mathrm{W}$ alcançando, portanto, a costa brasileira. Além dos sistemas meteorológicos citados, os quais interferem principalmente sobre as condições de precipitação, ventos e, consequentemente, altura de ondas, outros componentes atmosféricos podem prejudicar a navegação costeira pela diminuição da visibilidade, a exemplo da precipitação, como a presença de nevoeiros (Jin et al., 2001).

A análise do comportamento da velocidade do vento e altura de onda, que em geral tendem a aumentar em intensidade nas latitudes mais elevadas (Pimenta et al., 2008; Pianca et al., 2010), torna-se importante no contexto das ocorrências de naufrágios. Os ventos intensos podem provocar a inclinação da embarcação até um ângulo onde ocorra o inundamento ou tombamento ocasionado pelas ondas, o que pode contribuir para a ocorrência de naufrágio, somado ou não a outros fatores como falta de manutenção ou imperícia na condução. Ventos intensos e persistentes sobre o oceano são responsáveis pela elevação na altura das ondas, tornando as condições de mar muito perigosas para atividades de navegação (Soares et al., 2001).

Esse estudo pretende analisar o comportamento da velocidade do vento e da altura de onda durante os incidentes de naufrágio registrados na costa brasileira, entre os estados do Sergipe e do Rio Grande do Sul, com o objetivo de identificar, caso a caso, qual a contribuição desses fatores para a ocorrência dos mesmos. Os resultados encontrados apontaram maior incidência de naufrágios nas regiões sudeste e sul do Brasil, com maior ocorrência entre os meses de março e agosto, envolvendo principalmente embarcações de pequeno porte. Embora reconhecendo a importância de outras variáveis meteorológicas para a ocorrência de naufrágios, neste estudo foi abordado apenas o comportamento e a climatologia da velocidade de vento e altura de ondas, bem como, a ocorrência de sistemas meteorológicos relacionados a esses fatores.

\section{MATERIAL E MÉTODOS}

Os dados de naufrágios foram cedidos pelo Serviço de Busca e Salvamento da Marinha (SALVAMAR), sendo referentes às áreas (Distritos Navais), datas da ocorrência, distâncias da costa e peso das embarcações. As distâncias, em milhas náuticas $(1$ milha náutica $=1$ minuto $=1852 \mathrm{~m})$ foram classificadas em intervalos de 0 a 12', 12 a 50', 50 a 200' e acima de 200'. O peso das embarcações, em toneladas, foi classificado de 0 a 1000 , de 1000 a 10000, e acima de 10000 ton. Os dados se referem, exclusivamente, a naufrágios ocorridos nos $1^{\circ}$, $2^{\circ}$ e $5^{\circ}$ Distritos Navais (DN), no período entre janeiro/2004 e junho/2008. Não foram disponibilizadas as coordenadas geográficas (latitude e longitude) ou informações sobre possíveis causas dos incidentes. A Figura 1 ilustra a área de abrangência dos distritos navais. 


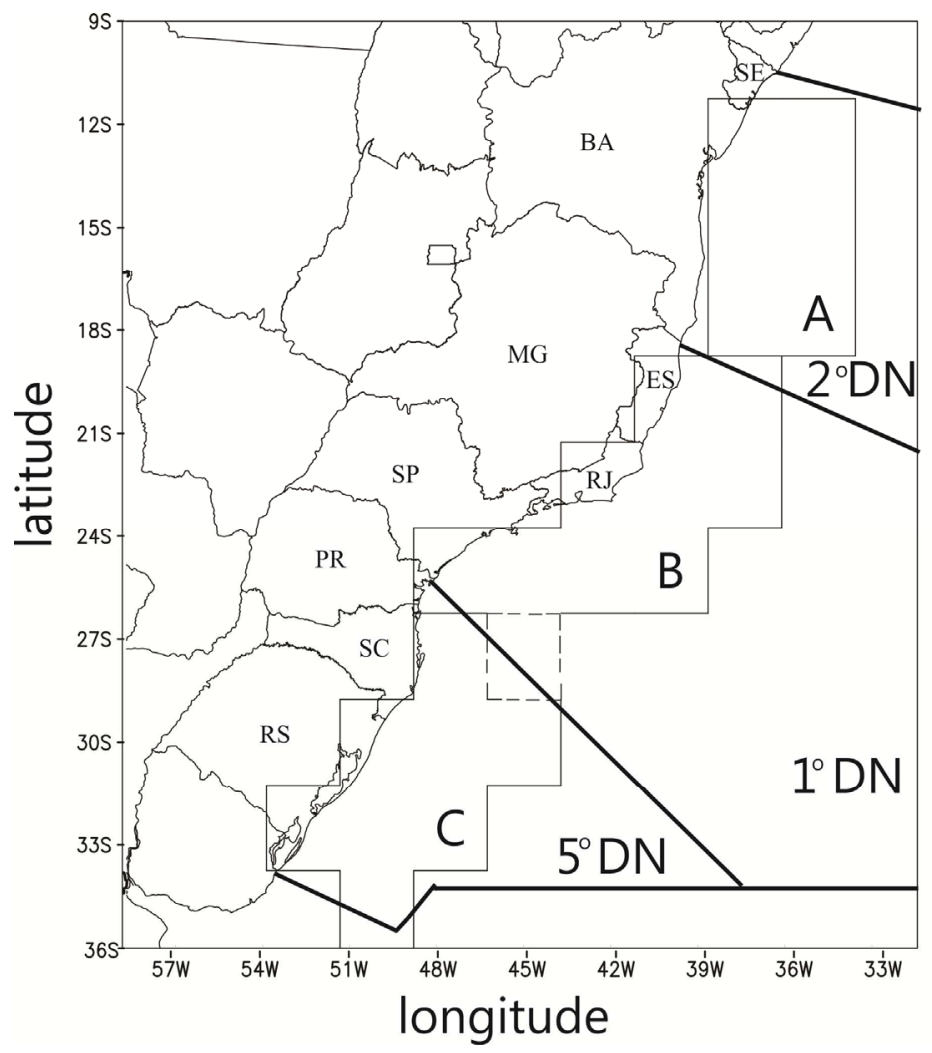

Figura 1 - Ambientes costeiros abrangidos pelos $1^{\circ}, 2^{\circ}$ e $5^{\circ}$ Distritos Navais (DN), delimitados no oceano pela linha grossa. As áreas A, B e C são representativas dos $\mathrm{DN}$ e indicam o domínio espacial utilizado para obtenção dos dados de vento e onda. A pequena área delimitada por linha tracejada faz parte de ambas as áreas C e B.

Os dados de vento foram obtidos da reanálise do National Center for Environmental Prediction / National Center for Atmospheric Research (NCEP/NCAR), que possui resolução espacial de $2,5^{\circ}$ de latitude e longitude e resolução temporal de $6 \mathrm{~h}(0000,0600,1200$ e 1800 UTC) (Kalnay et al., 1996). Os dados de altura significativa de onda foram obtidos do modelo Wave Watch III disponibilizado pelo National Oceanic and Atmospheric Administration / National Center for Environmental Prediction (NOAA/NCEP) (Tolman, 1999). Esses dados possuem resolução espacial de $0,5^{\circ}$ de latitude e longitude e resolução temporal de $3 \mathrm{~h}(0000,0300,0600,0900$, $1200,1500,1800$ e 2100 UTC).

As velocidades do vento $\left(\mathrm{m} \mathrm{s}^{-1}\right)$ foram calculadas a partir das componentes zonal $(u)$ e meridional $(v)$, no nível isobárico de $1000 \mathrm{hPa}$ da reanálise do NCEP/NCAR. A velocidade média $\left(V_{\mathrm{MED}}\right)$ foi calculada pela média espacial das áreas representantes de cada DN e média temporal dos horários 0000, 0600, 1200 e 1800 UTC. A velocidade máxima $\left(V_{\mathrm{MAX}}\right)$ é o valor máximo em cada área e entre os horários das 0000, 0600, 1200 e 1800 UTC. A altura significativa de onda $(\mathrm{m})$ foi calculada pela média espacial das áreas representantes de cada DN e média temporal dos horários 0000, 0300, 0600, 0900, 1200, 1500, 1800 e 2100
UTC. Os resultados de altura significativa das ondas foram subtraídos de seu valor climatológico (específica para cada área representante dos $\mathrm{DN}^{\prime}$ s) para a obtenção da anomalia da altura significativa $(A O)$. Optou-se por utilizar a climatologia mensal e não a climatologia diária da altura de onda devido ao período de dados ser de apenas sete anos (2005-2011). Para os casos de naufrágios ocorridos em 2004 e janeiro de 2005, a análise de onda não foi realizada devido à indisponibilidade de dados.

A avaliação das condições meteorológicas e oceânicas nos três DN foi divida em etapas. Primeiramente, para cada caso de naufrágio, foram analisados os campos meteorológicos médios diários de pressão ao nível médio do mar (reanálise NCEP/NCAR), além de imagens dos satélites GOES 12 e 10, no canal infravermelho, fornecidas pelo Centro de Previsão do Tempo e Estudos Climáticos / Instituto Nacional de Pesquisas Espaciais (CPTEC/INPE), no intuito de analisar subjetivamente a ocorrência de sistemas meteorológicos de escala sinótica na ocasião dos naufrágios. Posteriormente, foram feitas as análises objetivas com base em valores médios e máximos de cada área, devido a não disponibilidade do georreferenciamento exato (latitude e longitude) dos locais de ocorrência dos naufrágios. As frequências mensais e trimestrais dos incidentes foram 
comparadas, respectivamente, com a climatologia do vento mensal e por estação do ano. Para cada data e DN de ocorrência de naufrágio, a $V_{\mathrm{MED}}, V_{\mathrm{MAX}}$ e $A O$ foram utilizadas.

Os resultados da velocidade do vento foram comparados à média climatológica diária, desvio e valores mínimos e máximos históricos da mesma data e local, extraídos da série histórica de 1957 a 2008 da reanálise do NCEP/NCAR. Os valores de $V_{\text {MAX }}$ foram avaliados segundo a classificação de Beaufort (Byers, 1944), que se subdivide em 13 classes, que variam de força 0 (calmaria) a força 12 (furacão). As condições do mar foram avaliadas com base nos valores de $A O$.

\section{RESULTADOS E DISCUSSÃO}

Foram registradas 69 ocorrências de naufrágio durante o período estudado, sendo $94 \%$ referentes a embarcações de até 1000 ton. O restante envolveu embarcações de 1000 a 10000 ton. Com relação às distâncias da costa, $68 \%$ ocorreram até 12 ', $20 \%$ entre 12 e $50^{\prime}$ e $12 \%$ entre 50 e 200 '. As datas, peso das embarcações e distância da costa de cada caso registrados nos $1^{\circ}, 2^{\circ}$ e $5^{\circ} \mathrm{DN}$ são apresentadas na Tabela 1.

A análise dos campos meteorológicos e das imagens de satélite identificou quatro tipos de sistemas de escala sinótica, capazes de gerar ventos mais intensos sobre as áreas de ocorrência de naufrágios. Em 56\% dos casos, foi identificada a presença de frentes frias, em 19\%, foi identificada ciclogênese em superfície, em $12 \%$, foi verificado intenso gradiente horizontal de pressão em superfície, provocado pela atuação de sistemas de alta e baixa pressão. No restante dos casos, foram verificados sistemas pouco favoráveis a geração de ventos, como os sistemas de alta pressão com centro sobre a área do naufrágio, ou ausência de sistema meteorológico significativo dentro do domínio sinótico. Em 28/março/2004, foi registrado o primeiro furacão da história no oceano Atlântico Sul, no $5^{\circ}$ DN (Pezza e Simmonds, 2005), sendo registrado um naufrágio associado ao evento neste distrito (Tabela 1).

Alguns sistemas meteorológicos podem gerar ventos com intensidade suficiente para a formação de ondas elevadas, e estas se propagarem por grandes distâncias com pouca atenuação (Melo Filho, 1993, 2004; Campos et al., 2010). Em eventos extremos, há ondas que podem percorrer distâncias comparáveis à metade da circunferência da Terra (Barber e Ursell, 1948). Soares et al. (2001) estudaram a distribuição dos acidentes com embarcações e sua relação com as condições severas de tempo no Atlântico Norte, com base na análise de campos climatológicos de altura de onda, demonstrando que a frequência de acidentes esteve relacionada com uma elevada altura de onda significativa.

No presente estudo, a maioria dos naufrágios envolveu embarcações de pequeno porte, em dias e áreas sob atuação de sistemas meteorológicos. Assim, pode-se considerar que, direta ou indiretamente, esses acidentes tiveram forte relação com as condições de tempo e/ou mar.

\subsection{Avaliação trimestral e mensal}

A frequência de naufrágios na costa brasileira foi maior entre março e agosto, e relativamente menor em dezembro, janeiro e fevereiro (Figura 2). Padrão similar foi observado para os naufrágios ocorridos no $1^{\circ}$ e $5^{\circ} \mathrm{DN}$. O aumento na frequência de naufrágios durante determinada época do ano pode estar relacionado a diversos fatores, como por exemplo, o aumento na quantidade de embarcações em atividade pesqueira, durante períodos propícios. Entretanto, com relação ao fator climático, a

Tabela 1 - Datas*, peso* (P) das embarcações (em toneladas), distância* (D) da costa (em milhas náuticas) e sistemas meteorológicos em escala sinótica observados nos naufrágios $(\mathrm{N})$ ocorridos nos $1^{\circ}, 2^{\circ}$ e $5^{\circ}$ Distritos Navais $(\mathrm{DN})$ * $^{*}$ - Fonte: SALVAMAR

\begin{tabular}{|c|c|c|c|c|c|c|c|c|c|c|c|c|c|c|c|c|c|c|c|c|c|c|c|c|c|c|c|c|c|}
\hline & $1^{\circ} \mathrm{DN}$ & & & & & $1^{\circ} \mathrm{DN}$ & & & & & $1^{\circ} \mathrm{DN}$ & & & & & $2^{\circ} \mathrm{DN}$ & & & & & $5^{\circ} \mathrm{DN}$ & & & & & $5^{\circ} \mathrm{DN}$ & & & \\
\hline $\mathbf{N}$ & Data & $\mathbf{P}$ & D & $\mathbf{S}$ & $\mathbf{N}$ & Data & $\mathbf{P}$ & D & $\mathbf{S}$ & $\mathbf{N}$ & Data & $\mathbf{P}$ & D & $\mathbf{S}$ & $\mathbf{N}$ & Data & $\mathbf{P}$ & D & $\mathbf{S}$ & $\mathbf{N}$ & Data & $\mathbf{P}$ & D & $\mathbf{S}$ & $\mathbf{N}$ & Data & $\mathbf{P}$ & D & $\mathbf{S}$ \\
\hline 1 & $13 / 03 / 2004$ & A & 1 & ff & 13 & $02 / 07 / 2006$ & $\mathrm{~A}$ & 1 & $\mathrm{cs}$ & 25 & $02 / 11 / 2007$ & A & 3 & $\mathrm{ff}$ & 3 & $11 / 09 / 2005$ & A & 1 & as & 7 & $21 / 07 / 2004$ & A & 1 & gp & 19 & $09 / 05 / 2007$ & A & 1 & gp \\
\hline 2 & $06 / 05 / 2004$ & $\mathrm{~A}$ & 3 & cs & 14 & $12 / 08 / 2006$ & $\mathrm{~A}$ & 2 & cs & 26 & $23 / 11 / 2007$ & A & 2 & $\mathrm{ff}$ & 4 & $11 / 10 / 2005$ & B & 1 & as & 8 & $01 / 01 / 2005$ & $\mathrm{~A}$ & 2 & as & 20 & $01 / 06 / 2007$ & A & 1 & $\mathrm{ff}$ \\
\hline 3 & $04 / 07 / 2004$ & A & 1 & $\mathrm{ff}$ & 15 & $15 / 08 / 2006$ & $\mathrm{~A}$ & 2 & $\mathrm{ff}$ & 27 & $25 / 11 / 2007$ & A & 1 & ff & 5 & $14 / 07 / 2006$ & A & 1 & cs & 9 & $23 / 01 / 2005$ & A & 1 & $\mathrm{ff}$ & 21 & $28 / 06 / 2007$ & A & 1 & cs \\
\hline 4 & $08 / 10 / 2004$ & A & 2 & cs & 16 & $24 / 09 / 2006$ & A & 1 & $\mathrm{ff}$ & 28 & $04 / 12 / 2007$ & A & 3 & $\mathrm{ff}$ & 6 & $19 / 06 / 2008$ & $\mathrm{~B}$ & 2 & $\mathrm{cs}$ & 10 & $20 / 03 / 2005$ & A & 1 & $\mathrm{ff}$ & 22 & $11 / 09 / 2007$ & A & 1 & $\mathrm{ff}$ \\
\hline 5 & $11 / 04 / 2005$ & $\mathrm{~B}$ & 1 & cs & 17 & $25 / 09 / 2006$ & $\mathrm{~A}$ & 1 & $\mathrm{ff}$ & 29 & $25 / 03 / 2008$ & A & 1 & $\mathrm{ff}$ & & $5^{\circ} \mathrm{DN}$ & & & & 11 & 06/07/2005 & A & 1 & cs & 23 & $17 / 11 / 2007$ & A & 1 & as \\
\hline 6 & $26 / 04 / 2005$ & A & 1 & $\mathrm{ff}$ & 18 & $16 / 11 / 2006$ & $\mathrm{~A}$ & 2 & cs & 30 & $30 / 03 / 2008$ & A & 1 & $\mathrm{ff}$ & $\mathbf{N}$ & Data & $\mathbf{P}$ & D & $\mathbf{S}$ & 12 & $15 / 07 / 2005$ & A & 3 & $\mathrm{ff}$ & 24 & $29 / 12 / 2007$ & A & 1 & $\mathrm{ff}$ \\
\hline 7 & $23 / 08 / 2005$ & A & 1 & as & 19 & $10 / 03 / 2007$ & A & 1 & $\mathrm{ff}$ & 31 & $16 / 04 / 2008$ & A & 1 & $\mathrm{ff}$ & 1 & $28 / 03 / 2004$ & A & 3 & fc & 13 & $07 / 08 / 2005$ & A & 2 & $\mathrm{ff}$ & 25 & $08 / 01 / 2008$ & A & 1 & as \\
\hline 8 & $13 / 11 / 2005$ & A & 1 & $\mathrm{ff}$ & 20 & $06 / 05 / 2007$ & A & 1 & cs & 32 & $16 / 06 / 2008$ & A & 2 & ff & 2 & $25 / 04 / 2004$ & A & 3 & gp & 14 & $30 / 09 / 2005$ & A & 1 & $\mathrm{ff}$ & 26 & $20 / 01 / 2008$ & A & 1 & $\mathrm{ff}$ \\
\hline 9 & $26 / 01 / 2006$ & A & 1 & $\mathrm{ff}$ & 21 & $16 / 06 / 2007$ & B & 1 & $\mathrm{ff}$ & & $2^{\circ} \mathrm{DN}$ & & & & 3 & $21 / 05 / 2004$ & A & 1 & gp & 15 & $05 / 09 / 2006$ & A & 2 & gp & 27 & $15 / 04 / 2008$ & A & 1 & gp \\
\hline 10 & $05 / 03 / 2006$ & A & 2 & $\mathrm{ff}$ & $22^{\#}$ & $06 / 08 / 2007$ & A & 1 & $\mathrm{ff}$ & $\mathbf{N}$ & Data & $\mathbf{P}$ & D & $\mathbf{S}$ & 4 & $07 / 06 / 2004$ & A & 2 & $\mathrm{ff}$ & 16 & $10 / 09 / 2006$ & A & 1 & $\mathrm{ff}$ & 28 & $21 / 04 / 2008$ & A & 1 & cs \\
\hline 11 & $10 / 05 / 2006$ & A & 1 & cs & 23 & 04/09/2007 & $\mathrm{A}$ & 1 & as & 1 & $13 / 01 / 2005$ & A & 1 & ff & 5 & $15 / 06 / 2004$ & A & 1 & as & 17 & $18 / 09 / 2006$ & A & 2 & $\mathrm{ff}$ & 29 & $28 / 05 / 2008$ & A & 1 & $\mathrm{ff}$ \\
\hline 12 & $29 / 05 / 2006$ & A & 3 & gp & 24 & $25 / 09 / 2007$ & A & 1 & $\mathrm{ff}$ & 2 & $09 / 07 / 2005$ & A & 2 & $\mathrm{ff}$ & 6 & $01 / 09 / 2004$ & A & 3 & as & 18 & $13 / 04 / 2007$ & $\mathrm{~A}$ & 1 & $\mathrm{ff}$ & 30 & $22 / 06 / 2008$ & A & 1 & gp \\
\hline
\end{tabular}




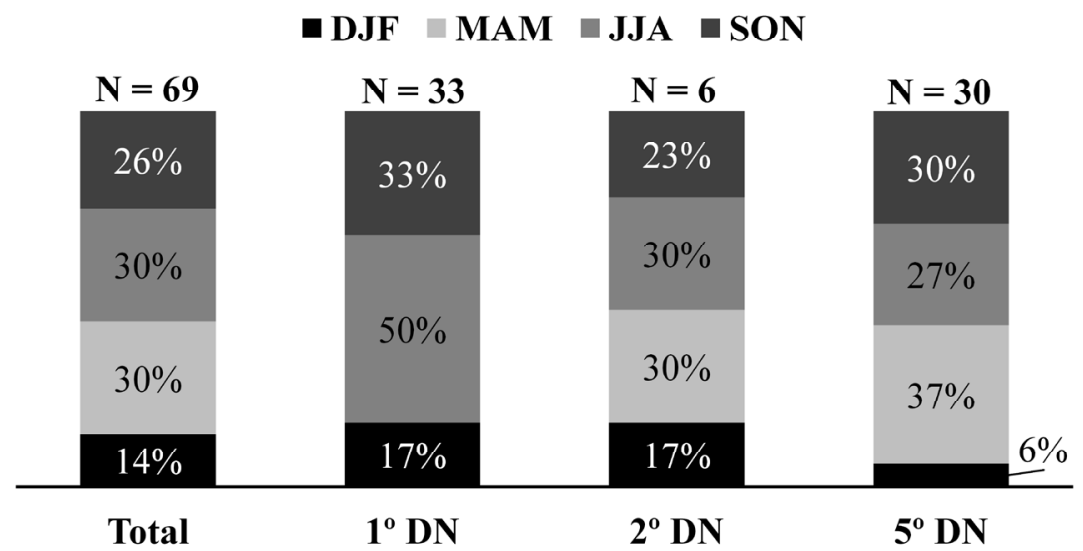

Figura 2 - Frequência trimestral de naufrágios para a área total de estudo, $1^{\circ}, 2^{\circ}$ e $5^{\circ}$ Distritos Navais (DN). N = número de ocorrências. DJF dezembro/janeiro/fevereiro; MAM - março/abril/maio; JJA - junho/julho/agosto; SON - setembro/outubro/novembro.

região do $5^{\circ} \mathrm{DN}$ possui climatologia de eventos meteorológicos mais intensos em meses com temperaturas mais baixas. Os ciclones extratropicais que atuam nessa área oceânica, por exemplo, são mais frequentes e intensos no inverno (Gan e Rao, 1991; Sinclair, 1994; 1995). O baixo número de naufrágios (N $=6$ ) e menor variabilidade climática ao longo do ano no $2^{\circ} \mathrm{DN}$ não permitem suposições sobre associações dessas ocorrências com as estações do ano (Figura 2).

A variabilidade histórica da velocidade do vento mostrou tendências similares entre os DN (Figura 3). No entanto, os menores valores no $1^{\circ}$ e $2^{\circ} \mathrm{DN}$ ocorreram entre março e abril, enquanto que no $5^{\circ} \mathrm{DN}$ estes ocorreram entre fevereiro e março. Por outro lado, o pico máximo da climatologia mensal do vento ocorreu em setembro no $1^{\circ}$ e $5^{\circ} \mathrm{DN}$, e em julho no $2^{\circ}$ DN. A variabilidade histórica da altura das ondas apresentou a mesma tendência da climatologia do vento nos $2^{\circ}$ e $5^{\circ} \mathrm{DN}$, indicando que as ondas estiveram bem relacionadas com os sistemas meteorológicos atuantes nessas regiões. Por outro lado, essa coincidência não foi verificada no $1^{\circ} \mathrm{DN}$, entre março e setembro. Nesse período, correspondente ao outono e inverno austral, a variabilidade da altura das ondas nesta região coincidiu claramente com a climatologia do vento e das ondas no $5^{\circ} \mathrm{DN}$, indicando que as mesmas podem ter sido geradas por sistemas meteorológicos atuantes em outras áreas, tais como ciclones extratropicais atuantes no oceano, próximos a região Sul do Brasil.

$\mathrm{O} 2^{\circ} \mathrm{DN}$ foi a região onde a frequência de naufrágios mais coincidiu com a climatologia do vento e das ondas, tendo a maioria ocorrido em julho, o período de ventos mais intensos. Não foram registradas ocorrências nos meses com menores velocidades do vento, ressaltando-se a baixa incidência de naufrágios no $2^{\circ} \mathrm{DN}$. No $1^{\circ} \mathrm{DN}$, foi verificada fraca relação entre o vento climatológico e a frequência mensal de naufrágios, com incidência de casos tanto em meses de vento forte quanto em meses de vento fraco. Por outro lado, salvo poucas exceções, no $5^{\circ} \mathrm{DN}$, observou-se clara tendência de aumento (ou diminuição) da frequência de naufrágio coincidente com aumento (ou diminuição) da velocidade climatológica do vento,

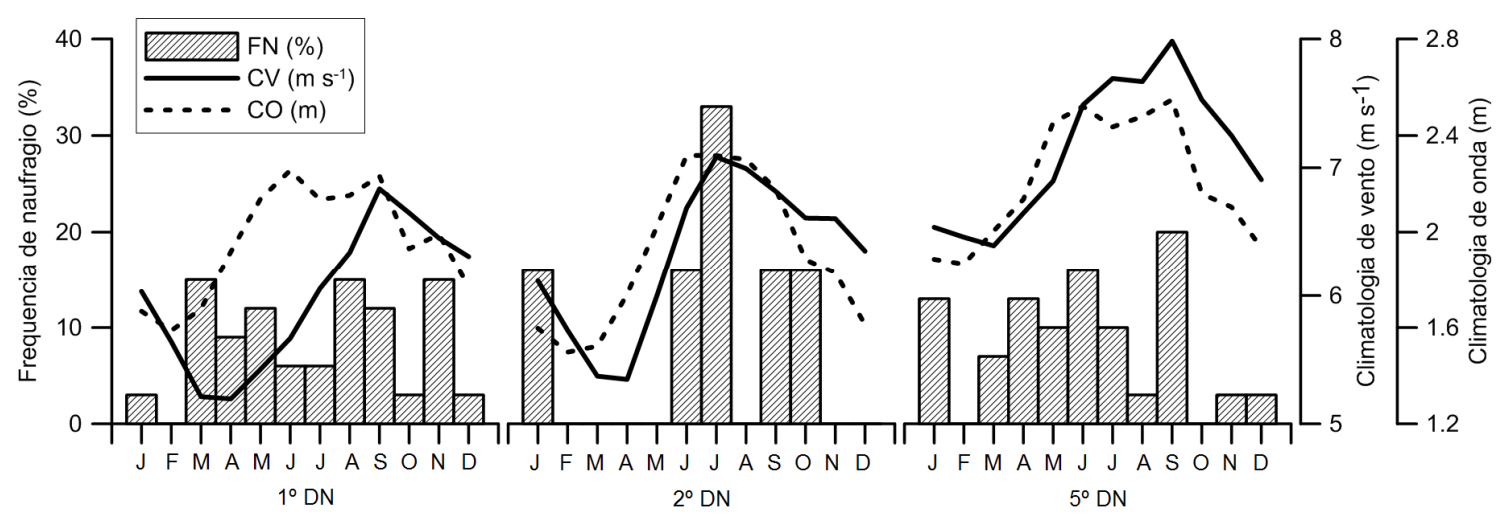

Figura 3 - Frequência mensal de naufrágios $(\mathrm{FN})$ para o $1^{\circ}, 2^{\circ}$ e $5^{\circ}$ Distritos Navais (DN) e climatologia mensal da velocidade do vento (CV) e da altura significativa de onda (CO). 
bem como da altura climatológica das ondas ao longo do ano. As exceções podem estar relacionadas a outros fatores, como períodos de atividade pesqueira intensa, conforme anteriormente mencionado, ou com outros fatores não relacionados às condições meteorológicas.

\subsection{Avaliação dos ventos e das alturas de onda para cada caso de naufrágio}

As Figuras 4, 5 e 6 mostram a comparação do $V_{\mathrm{MED}} \mathrm{e}$ $V_{\text {MAX }}$ do dia de ocorrência do naufrágio com os parâmetros estatísticos obtidos da reanálise do período de 1957 a 2008, assim como da análise da anomalia de altura de onda.

No $1^{\circ} \mathrm{DN}$, o $V_{\mathrm{MED}}$ no dia do naufrágio ficou acima do $V_{\mathrm{MED}}$ climatológico em 20 dos 32 casos (Figura 4a). Entre os casos com anomalia positiva, o $V_{\mathrm{MED}}$ ultrapassou o desvio padrão em sete deles, indicando que o vento foi muito forte nessas ocasiões. Por vezes, o $V_{\mathrm{MED}}$ foi tão intenso que chegou próximo ao valor máximo histórico (caso 18, Figura 4a). Por outro lado, em 10 casos de naufrágio o $V_{\mathrm{MED}}$ ficou abaixo do $V_{\mathrm{MED}}$ climatológico, e em dois casos de naufrágio o $V_{\mathrm{MED}}$ foi igual ou muito próximo ao $V_{\mathrm{MED}}$ climatológico. A $A O$ (Figura 4c) mostrou que a altura de onda foi superior à média climatológica em 13 e inferior em 12 dos 29 casos analisados. Em três casos, a altura de onda esteve dentro da normalidade. Nos casos onde a $V_{\text {MED }}$ do naufrágio foi abaixo da climatologia (casos 11, 17, 19, 22, 26, 27, 28 e 29), um dos casos (11) apresentou altura de onda normal, quatro $(19,26,27$ e 29) acima e três $(17,22$ e 28$)$ abaixo do normal. Por outro lado, nos casos onde os dados estão disponíveis, nas 18 vezes que o $V_{\mathrm{MED}}$ do naufrágio esteve acima da climatológica, a altura de onda ficou acima do normal na metade desses casos, abaixo em seis casos e dentro da normalidade em três casos.

Os casos com ausência de ventos fortes e presença de ondas elevadas podem ter sido originados pela atuação de sistemas meteorológicos ocorridos à distância, os quais podem gerar ondas de plataforma continental (Melo Filho, 1993, 2004; Castro e Lee, 1995; Campos et al., 2010), alcançando regiões onde a velocidade do vento se encontra em condições normais. Em estudo na costa sudeste do Brasil (Castro e Lee, 1995) foi observado que flutuações do nível do mar, registradas em porções do centro ao norte da região estudada, estiveram mais bem correlacionadas com ventos registrados mais ao sul e em períodos anteriores de tempo. Apenas três casos (17, 22 e 28) ocorreram em condições de ventos e altura de ondas abaixo da climatologia, indicando que esses fatores não foram as prováveis causas desses naufrágios.

$\mathrm{O} V_{\text {MAX }}$ ficou acima da climatologia em 17 dos 32 casos de naufrágio (Figura 4b). Entre os casos de anomalia positiva, o $V_{\mathrm{MAX}}$ foi superior ao desvio padrão em sete deles, e em cinco
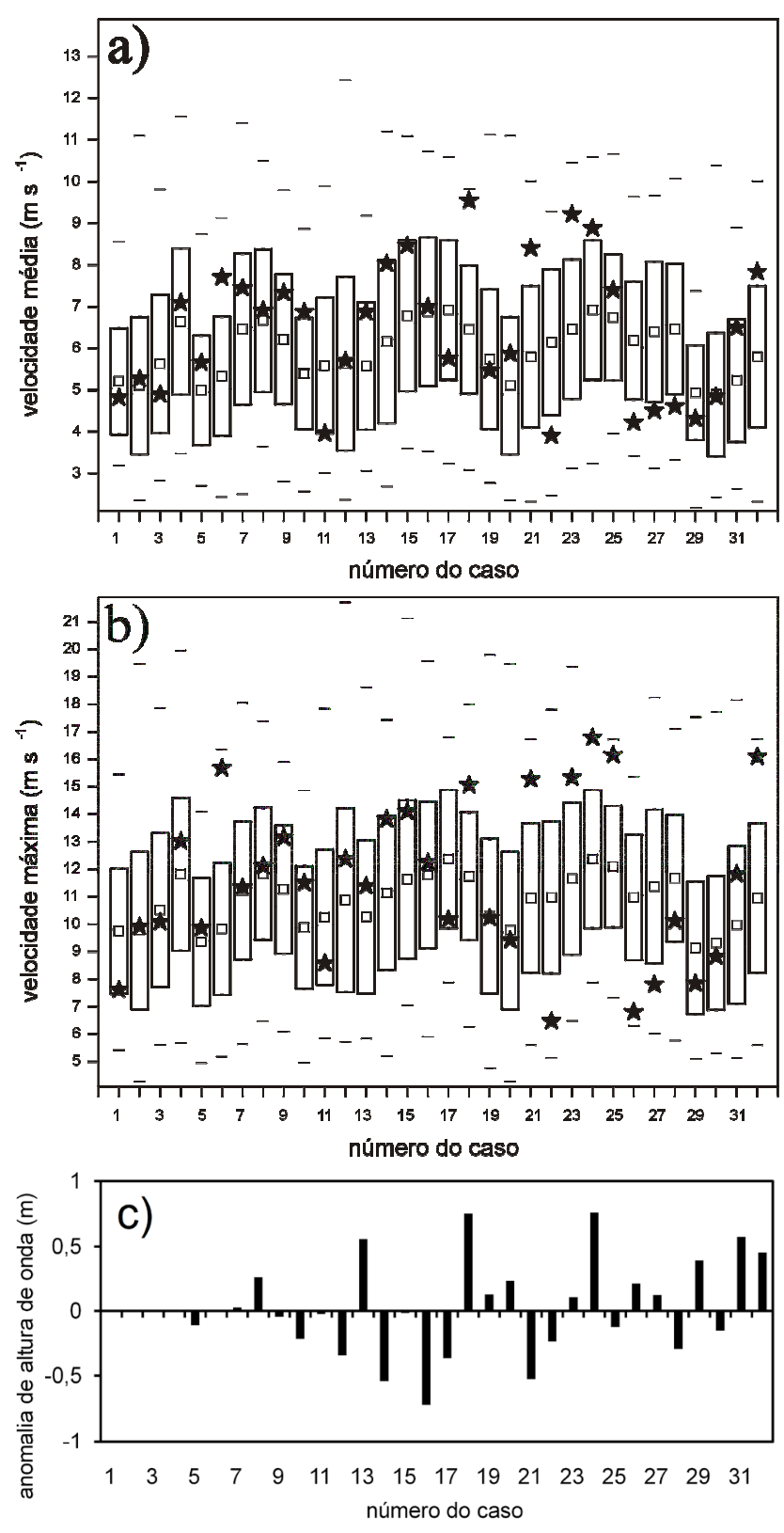

Figura 4 - Resultados para o $1^{\circ}$ Distrito Naval. (a) Velocidade do vento média $\left(V_{\mathrm{MED}}\right)$, (b) Velocidade do vento máxima $\left(V_{\mathrm{MAX}}\right)$ e (c) Anomalia da altura de onda significativa $(A O)$. A estrela $(\star)$ refere-se ao valor calculado para o dia do naufrágio, o quadrado $(\square)$ refere-se à média climatológica e a barra cobre o intervalo $\pm \sigma$ (desvio padrão). Os símbolos (-) referem-se aos valores máximos e mínimos da série histórica. A $A O$ dos quatro primeiros casos não foram avaliados por falta de dados de onda.

casos $\left(6,21,24,25\right.$ e 32) o valor de $V_{\mathrm{MAX}}$ foi próximo ou igual ao recorde de vento máximo na área. Para os 15 demais, o $V_{\mathrm{MAX}}$ esteve abaixo (10 casos) ou igual ao valor climatológico (cinco casos). Segundo a classificação de Beaufort (Byers, 1944), em nove casos o $V_{\mathrm{MAX}}$ atingiu força 6 e em outros nove casos o 
$V_{\text {MAX }}$ atingiu força 7 . De acordo com essa classificação, o estado do mar seria de "grandes vagas, cristas espumosas brancas e borrifos" com a força 6 e de "vagalhões pequenos com espumas em faixas" com a força 7.

Em 6/agosto/2007 (caso 22), ocorreram dois naufrágios, tendo a $A O$, o $V_{\text {MED }}$ e $V_{\text {MAX }}$ ficado abaixo da climatologia e, com ventos abaixo do desvio padrão. Apesar disso, as análises dos campos meteorológicos e das imagens de satélite indicaram a presença de uma frente fria. Da mesma forma, dois naufrágios ocorreram entre 24 e 25/setembro/2006, no $1^{\circ} \mathrm{DN}$ (casos 16 e 17, Figura 4). O $V_{\text {MED }}$ e o $V_{\text {MAX }}$ ficaram próximo da média climatológica em 24/setembro e abaixo dela em 25/setembro. A altura de onda esteve abaixo da climatologia nos dois dias. E, novamente, a avaliação meteorológica subjetiva mostra uma frente fria atuando sobre o $1^{\circ} \mathrm{DN}$ durante esses dois dias.

É importante ressaltar que os campos meteorológicos gerados por modelos numéricos, como a reanálise do NCEP/ NCAR, são mais adequados para identificação dos sistemas meteorológicos atuantes sobre um domínio sinótico, do que para obtenção de valores pontuais das variáveis meteorológicas. As resoluções espaciais e temporais e as técnicas de assimilação de dados utilizadas para a construção da reanálise podem dificultar a detecção de determinados eventos atmosféricos, acarretando em subestimação dos valores de vento e, consequentemente, dos valores de altura de onda.

Entre os naufrágios ocorridos no $2^{\circ} \mathrm{DN}$, o $V_{\mathrm{MED}}$ e $V_{\mathrm{MAX}}$ ficaram acima da climatologia em mais da metade dos casos (Figuras 5a e 5b). Nas quatro vezes em que o $V_{\text {MED }}$ superou a climatologia, superou também o desvio padrão, atingindo valor máximo histórico numa ocasião (caso 1) e ficando próximo do valor histórico em outra ocasião (caso 3 ). Para o caso 1 , o $V_{\text {MAX }}$ atingiu valor próximo do recorde histórico no $2^{\circ} \mathrm{DN}$, sendo este classificado como força 7, segundo a escala Beaufort. Outros três casos com $V_{\text {MAX }}$ acima da climatologia apresentaram força 6 . A $A O$ mostrou que, entre os cinco casos analisados (caso $1 \mathrm{sem}$ dados de onda), apenas um apresentou altura de onda abaixo da climatologia (Figura 5c).

$\mathrm{Na}$ avaliação do $V_{\mathrm{MED}}$ registrado no $5^{\circ} \mathrm{DN}$ (Figura 6a), em 14 casos o vento superou a climatologia. Entre os casos de anomalia positiva, nove ocorreram em dias que o $V_{\text {MED }}$ superou o desvio padrão. Em 11 casos, o $V_{\mathrm{MED}}$ registrado foi inferior à climatologia e em cinco casos, o $V_{\mathrm{MED}}$ apresentou valores muito próximos da climatologia.

Os resultados do $V_{\text {MAX }}$ (Figura 6b) foram similares. Em 15 dos 30 casos de naufrágio, o $V_{\text {MAX }}$ ficou acima da climatologia, superando o desvio padrão em quatro deles. Para a outra metade dos casos, em 13 ocorrências de naufrágio o registro de $V_{\text {MAX }}$ ficou abaixo da climatologia e em duas ocorrências o $V_{\text {MAX }}$ registrou o mesmo valor climatológico. Segundo a escala Beaufort, o $V_{\text {MAX }}$ atingiu força 6 em 12 casos e
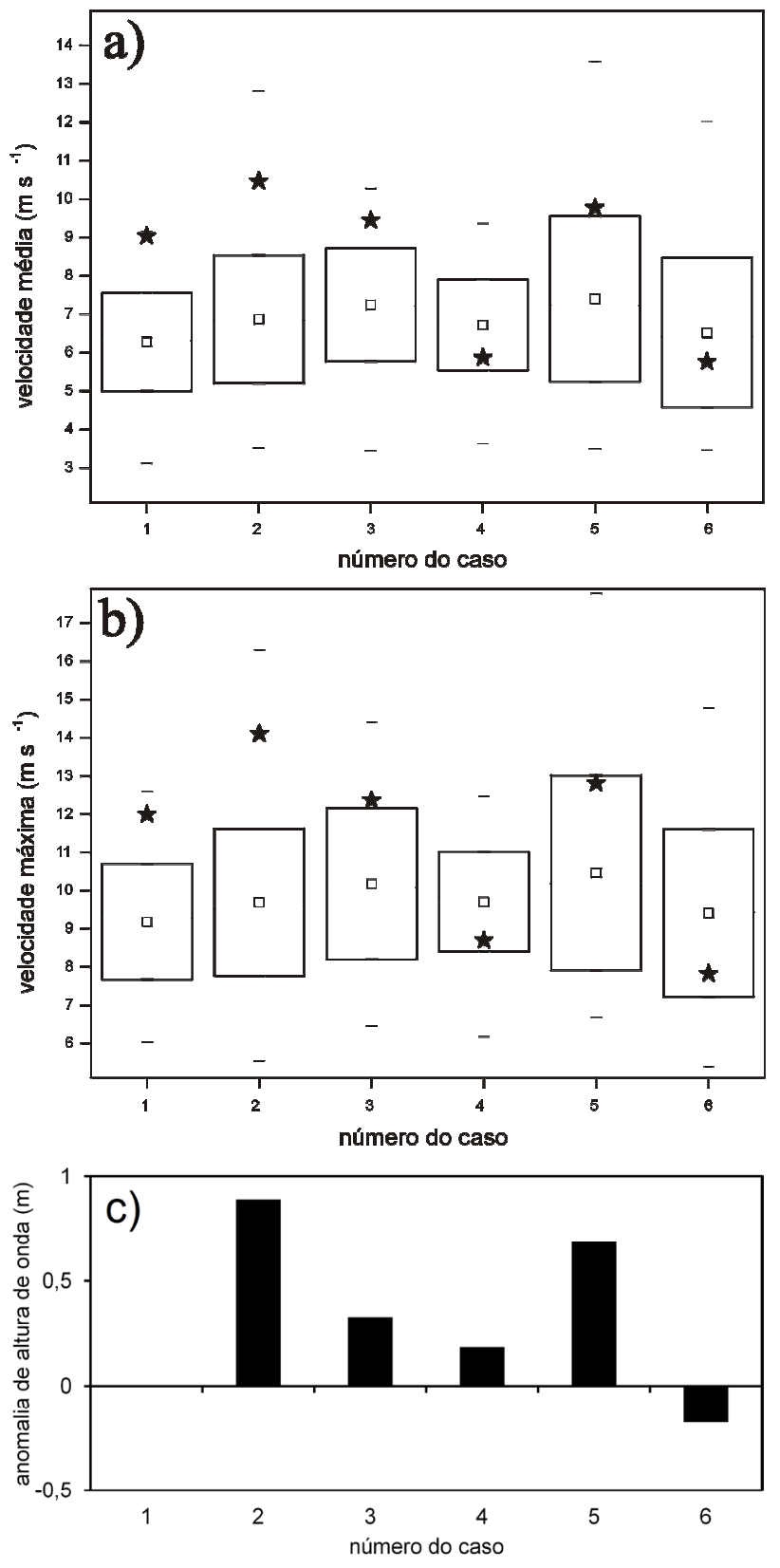

Figura 5 - Resultados para o $2^{\circ}$ Distrito Naval. (a) Velocidade do vento média $\left(V_{\mathrm{MED}}\right)$, (b) Velocidade do vento máxima $\left(V_{\mathrm{MAX}}\right)$ e (c) Anomalia da altura de onda significativa $(A O)$. A estrela $(\star)$ refere-se ao valor calculado para o dia do naufrágio, o quadrado $(\square)$ refere-se à média climatológica e a barra cobre o intervalo $\pm \sigma$ (desvio padrão). Os símbolos (-) referem-se aos valores máximos e mínimos da série histórica. A $A O$ do primeiro caso não foi avaliada por falta de dados de onda.

força 7 em 10 casos. Em um caso, referente ao naufrágio em 30/ setembro/2005, o valor do $V_{\text {MAX }}$ atingiu força 9 que, de acordo com a classificação Beaufort, favorece condições oceânicas de "vagalhões grandes a enormes e excepcionais, visibilidade reduzida a seriamente afetada". Apesar disso, a altura de onda 

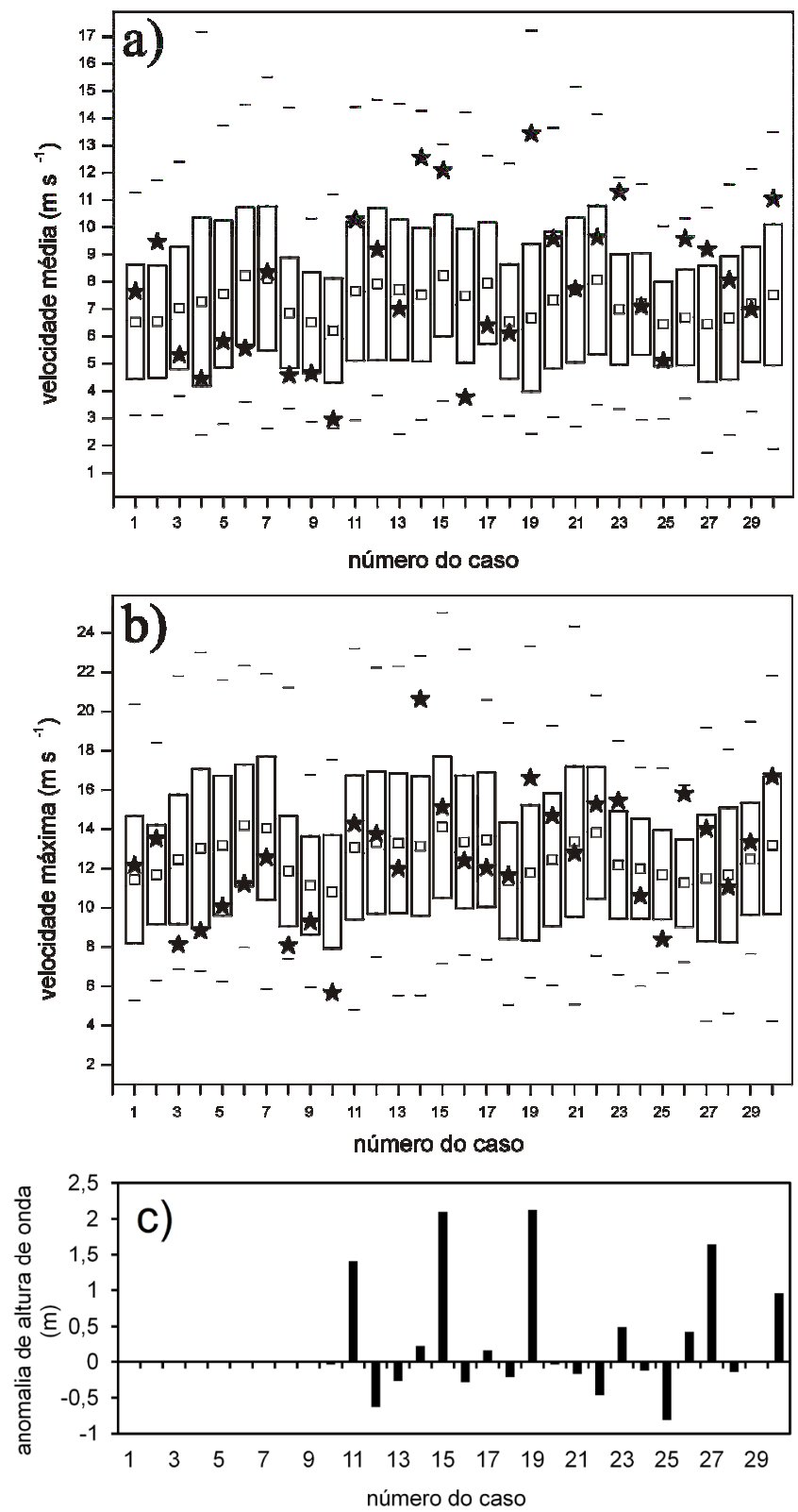

Figura 6 - Resultados para o $5^{\circ}$ Distrito Naval. (a) Velocidade do vento média $\left(V_{\mathrm{MED}}\right)$, (b) Velocidade do vento máxima $\left(V_{\mathrm{MAX}}\right)$ e (c) Anomalia da altura de onda significativa $(A O)$. A estrela $(\star)$ refere-se ao valor calculado para o dia do naufrágio, o quadrado $(\square)$ refere-se à média climatológica e a barra cobre o intervalo $\pm \sigma$ (desvio padrão). Os símbolos (-) referem-se aos valores máximos e mínimos da série histórica. A $A O$ dos nove primeiros casos não foram avaliadas por falta de dados de onda.

não apresentou valores extremos, embora tenha ficado acima da média climatológica (Figura 6c).

A $A O$ mostrou que em três casos de naufrágio a altura de onda ficou dentro da normalidade (Figura 6c). Para os demais, metade apresentou altura de onda acima e a outra metade abaixo da climatologia. Entretanto, os casos com
$A O$ positiva foram, em geral, mais significativos do que os casos com $A O$ negativa. Nos casos 11, 15, 19, 27 e 30, o mar apresentou-se com altura de onda próxima ou superior a $1 \mathrm{~m}$ acima da climatologia.

Na maioria das vezes em que as condições atmosféricas foram de ventos abaixo da climatologia, observou-se altura de ondas abaixo da normalidade. Apenas o caso do dia 18/ setembro/2006 (caso 17) apresentou altura de ondas levemente acima da média climatológica, indicando a possibilidade deste acidente ter ocorrido devido as condições oceânicas.

Destacaram-se os casos 15, 23 e 26, quando o $V_{\mathrm{MED}}$ registrou valores próximos do recorde (Figura 6a) e a $A O$ foi positiva (Figura 6c). Nessas ocasiões, foi registrada atuação de frente fria no $1^{\circ} \mathrm{DN}$, ao norte do $5^{\circ} \mathrm{DN}$. A passagem dessa frente fria foi seguida por um sistema de alta pressão relativamente intenso. E, devido ao gradiente horizontal de pressão observado, as condições atmosféricas tornam-se favoráveis para a ocorrência de ventos intensos. O $V_{\mathrm{MAX}}$ foi próximo do valor recorde no caso 26 (Figura 6 b).

$\mathrm{O} V_{\text {MED }}$ pode ser tratado como um parâmetro indicador de existência de vento abrangente em todo o DN e persistente durante parte do dia. Ventos abrangentes e persistentes são gerados por sistemas meteorológicos de escala sinótica, tais como, frentes frias ou ciclones extratropicais. Por outro lado, o $V_{\text {MAX }}$ pode ser tratado como um parâmetro indicador da intensidade desses sistemas meteorológicos, pois é calculado a partir do valor máximo na área e no dia. Dessa forma, nos momentos em que o $V_{\mathrm{MED}}$ e $V_{\mathrm{MAX}}$ foram maiores do que o desvio padrão acima da média, como os casos 6, 18, 21, 23, 24 e 32 do $1^{\circ} \mathrm{DN}$, casos 1,2 e 3 do $2^{\circ} \mathrm{DN}$ e casos $14,19,23$ e 26 do $5^{\circ} \mathrm{DN}$, pode-se supor que o sistema meteorológico atuante teve abrangência espacial, persistência temporal e intensidade. Entre esses casos de naufrágio, a maioria apresentou altura de onda acima do normal e $V_{\mathrm{MAX}}$ com classificação igual ou superior a força 7 .

\section{CONCLUSÕES}

As condições atmosféricas de escala sinótica, os valores médios e máximos da velocidade do vento e altura de onda em ambientes costeiros do Brasil, localizados ao sul da latitude de $10^{\circ} \mathrm{S}$, foram avaliados e relacionados aos casos de naufrágios ocorridos entre 2004 e 2008. A análise subjetiva dos campos meteorológicos e das imagens de satélite mostrou que, em apenas $12 \%$ dos casos, ocorreram condições meteorológicas pouco favoráveis à geração de ventos e ondas ou não existiu sistema no domínio sinótico. Na grande maioria dos casos, foi detectada a presença de sistemas meteorológicos, como frentes frias e ciclones extratropicais, nas áreas onde ocorreram os naufrágios ou em regiões próximas a estas. 
As comparações da climatologia do vento com a frequência mensal de naufrágios apontam algumas coincidências, como a tendência de aumento na frequência de casos nos meses onde, climatologicamente, o vento é mais intenso, conforme verificado no $2^{\circ}$ e $5^{\circ} \mathrm{DN}$. A análise dos ventos mostrou que em torno de $54 \%$ dos casos a velocidade média e máxima do vento apresentou-se superior à climatologia. Para a altura significativa de onda, os valores ficaram acima da normalidade em $46 \%$ dos casos de naufrágio. Os resultados apontaram maior incidência de naufrágios em ambientes costeiros das regiões sudeste e sul do Brasil, com maior ocorrência entre os meses de março e agosto, envolvendo principalmente embarcações de pequeno porte (94\%), utilizadas na pesca e navegação costeira.

Em vista dos resultados observados, torna-se evidente o importante papel das condições meteorológicas e oceanográficas sobre os incidentes de naufrágio na costa brasileira, principalmente em latitudes mais elevadas, onde os fenômenos atmosféricos são mais intensos. Estes resultados reforçam a importância da divulgação de informações às atividades de pesca e navegação costeira, em especial aquelas informações derivadas de boias de monitoramento e de sistemas de previsão do tempo e das ondas. Tais informações devem estar plenamente disponíveis, tanto durante o planejamento e início da atividade, quanto durante a permanência de embarcações em alto mar.

\section{AGRADECIMENTOS}

Os autores agradecem ao CNPq (processo $n^{\circ}$ 471917/2008-7) e FINEP (processo $n^{\circ}$ 2442008), pelo suporte financeiro, ao Comando de Operações Navais da Marinha do Brasil, pelos dados de naufrágios, ao CPTEC/INPE, pelas imagens de satélite, ao National Center for Environmental Prediction / National Center for Atmospheric Research (NCEP/NCAR), pelos dados da reanálise, ao National Oceanic and Atmospheric Administration / National Center for Environmental Prediction (NOAA/NCEP), pelos dados de altura significativa de onda, e aos revisores anônimos do sistema "peer review" da RBMET, pelas contribuições dadas para o melhoramento do manuscrito.

\section{REFERÊNCIAS BIBLIOGRÁFICAS}

BARBER, N. F.; URSELL, F. The generation and propagation of ocean waves and swell. I. Wave periods and velocities.

Philosophical Transactions of the Royal Society of London. Series A, Mathematical and Physical Sciences, v. 240 , p. 527-560, 1948.

BYERS, H. R. General meteorology. New York: McGraw-Hill Book Company, Inc, 1944.
CAMPOS, R. M.; CAMARGO, R.; HARARI, J. Caracterização de eventos extremos do nível do mar em Santos e sua correspondência com as reanálises do modelo NCEP no sudoeste do Atlântico Sul. Revista Brasileira de Meteorologia, v. 25, n. 2, p. 175-184, 2010.

CASTRO, B. M.; LEE, T. N. Wind-forced sea level variability on the southeast Brazilian shelf. Journal of Geophysical Research, v. 100, p. 16045-16056, 1995.

GAN, M. A.; RAO, V. B. Surface cyclogenesis over South America. Monthly Weather Review, v. 119, p. 1293-1302, 1991.

GARREAUD, R. D. Cold air incursions over Subtropical South America: mean structure and dynamics. Monthly Weather Review, v. 128, n. 7, p. 2544-2559, 2000.

JIN, D.; KITE-POWELL, H. L.; TALLEY, W. K. The safety of commercial fishing: determinants of vessel total losses and injuries. Journal of Safety Research, v. 32, p. 209228, 2001.

JIN, D.; KITE-POWELL, H. L.; THUNBERG, E.; SOLOW, A. R.; TALLEY, W. K. A model of fishing vessel accident probability. Journal of Safety Research, v. 33, p. 497-510, 2002.

JIN, D.; THUNBERG, E. An analysis of fishing vessel accidents in fishing areas off the northeastern United States. Safety Science, v. 43, p. 523-540, 2005.

KALNAY, E. et al. The NCEP/NCAR 40-Year Reanalysis Project. Bulletin of the American Meteorological Society, v. 77, n. 3, p. 437 - 471, 1996. LOIS, P.; WANG, J.; WALL, A.; RUXTON, T. Formal safety assessment of cruise ships. Tourism Management, v. 25, p. 93-109, 2002.

MELO FILHO, E. The Sea Sentinels project: watching waves in Brazil. In: Proceedings of $8^{\text {th }}$ Symposium on Coastal and Ocean Management (Coastal Zone) (pp. 505-517). American Society of Civil Engineerin, 1993.

MELO FILHO, E. Programa de Informação Costeira on-line: Um balanço dos primeiros dois anos de funcionamento. In: Seminario e Workshop em Engenharia Oceânica, 2004, Rio Grande. CD-ROM, 2004, v. 1, p. 1-16.

NEVES, M. A. S.; CARNEIRO, A. M. M.; PIMENTA, E. G. Análise estatística de acidentes com barcos de pesca. In: $1^{\circ}$ Congresso Nacional de Transportes Marítimos Construção Naval e Offshore (pp. 1-13). Rio de Janeiro: SOBENA, 2002.

PEZZA, A. B.; SIMMONDS, I. The first South Atlantic hurricane: unprecedented blocking, low shear and climate change. Geophysical Research Letters, v. 32, 2005.

PIANCA, C.; MAZZINI, P. L. F.; SIEGLE, E. Brazilian offshore wave climate based on NWW3 reanalysis. Brazilian Journal of Oceanography, v. 58, n. 1, p. 53-70, 2010. 
PIMENTA, F.; KEMPTON, W.; GARVINE, R. Combining meteorological stations and satellite data to avaluate the offshore wind power resource of Southeastern Brazil.

Renewable Energy, v. 33, n. 11, p. 2375-2387, 2008.

SATYAMURTY, P.; FERREIRA, C. D. C ; GAN, M. A. Cyclonic vortices over South America. Tellus, v. 1, n. 42, p. 194-201, 1990.

SIMMONDS, I.; KEAY, K. Mean Southern Hemisphere extratropical cyclone behavior in the 40-Year NCEP-NCAR Reanalysis. Journal of Climate, v. 13, p. 873-885, 2000.

SINCLAIR, M. R. An objective cyclone climatology for the Southern Hemisphere. Monthly Weather Review, v. 122, p. 2239-2256, 1994.

SINCLAIR, M. R. A climatology of cyclogenesis for the Southern Hemispheric. Monthly Weather Review, v. 123, p. 1601-1619, 1995.

SOARES, C. G.; BITNER-GREGERSEN, E.; ANTÃO, P. Analysis of the frequency of ship accidents under severe North Atlantic weather conditions. In: Proceedings of the Conference on Design and Operation for Abnormal Conditions II (RINA) (pp. 221-230). London, United Kingdom, 2001.
SOARES, C. G.; TEIXEIRA, A. P. Risk assessment in maritime transportation. Reliability Engineering and System Safety, v. 74, p. 299-309, 2001.

TOFFOLI, A.; LEFEVRE, J. M.; MONBALIU, J.; SAVINA, H.; BITNER-GREGERSEN, E. Freak waves: clues for predictions in ship accidents? In: Proceedings of the Thirteenth International Offshore and Polar Engineering Conference (pp. 23-29). Honolulu, USA, 2003.

TOLMAN, H. L. User manual and system documentation of WAVEWATCH III version 1.18. NOAA/NWS/NCEP/OMB Technical Note 166, 1999. 110 p. Disponível em <http:// polar.ncep.noaa.gov/waves/wavewatch>. Acesso em 02 Fev. 2012. 